\title{
Properties of CsI and CsI-TMAE Photocathodes
}

\author{
D.F. Anderson, S. Kwan and V. Peskov \\ Fermi National Accelerator Laboratory \\ P.O. Box 500, Batavia, Illinois 60510 \\ B. Hoeneisen \\ Universidad San Francisco de Quito \\ P.O. Box 841, Quito, Ecuador
}

June 1992

Presented at the International Conference on Advanced Technology and Particle Physics, Como, Italy, June 22-26, 1992

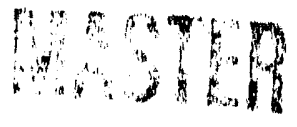




\section{Disclaimer}

This report was prepared as an account of work sponsored by an agency of the United States Government. Neither the United States Government nor any agency thereof, nor any of their employees, makes any warranty, express or implied, or assumes any legal liability or responsibility for the accuracy, completeness, or usefulness of any information, apparatus, product, ar process disclosed, or represents that its use would not infringe privately owned rights. Reference herein to any specific commercial product, process, or service by trade name, trademark, manufacturer, or otherwise, does not necessarily constitute or imply its endorsement, recommendation, or favoring by the United States Government or any agency thereof. The views and opinions of authors expressed herein do not necessarily state or reflect those of the United States Government or any agency thereof. 


\title{
Properties of CsI and CsI-TMAE Photocathodes
}

\author{
D.F. Anderson, S. Kwan, V. Peskov \\ Particle Detector Group \\ Fermi National Accelerator Laboratory \\ Batavia IL 60510 U.S.A. \\ and \\ B. Hoeneisen \\ Universidad San Francisco de Quito \\ P.O. Box 841, Suc. 12 Octubre \\ Quito, Ecuador
}

\begin{abstract}
The importance of heating the CsI or CSI-TMAE photocathodes during preparation, as well as the importance of the gas environment on the quantum efficiency is presented. The dependence of the aging characteristics of these photocathodes on the operating temperature, on the presence of gas, and on the charge amplification of the chamber is also discussed. For CsI photocathodes charges in excess of $2 \times 10^{14} \mathrm{e}^{-1 / \mathrm{mm}^{2}}$ can be collected with little degradation of performance. A timing resolution of $0.55 \mathrm{~ms}$ is also achieved for single photoelectrons suggesting a possible time-of-flight detector.
\end{abstract}




\section{Introduction}

For many years Ring Imaging Cherenkov detectors (RICH) have promised to be powerful toois for particle identification. But, up to now, the promise has not been fully fulfilled. RICH detectors using photosensitive gases (TEA and TMAE) have been slow and difficult to operate. Operation of TMAE gas in proportional counters has also shown to have severe aging problems [1]. The new enviromment of the SSC/LHC, RHIC, and the proposed B factories requinss detectors that can handle high rates and high multiplicities. These detectors must be stable for long periods of time under severe conditions.

New interest has developed in the field of RICH with the introduction of two solid photocathodes: CsI and CsI-TMAE (CSI with an adsorbed lay's of TMAE). These photocathodes have been shown by Séguinot et al. [2] to have remarkable quantum efficiencies (QE) of $35 \%$ and $46 \%$ a $170 \mathrm{~nm}$, respectively. These values were achieved by flowing pure methare over the photocathodes while they were maintained at $40^{\circ} \mathrm{C}$ [3]. The value of $35 \%$ for the CsI photocathode has not been confirmed in the literature. The high $\mathrm{QE}$ of the CsI-TMAE phntocathode has been achieved [4] using a technique of increasing the QE by extracting charge from the photocathode. Measurements employing more conventional techniques have yielded a somewhat lower QE [5-8]. In this work we confirm the results of Séguinot et al. for the CsI and CsI-TMAE photocathodes.

Charpak et al. [5] first suggested depositing the photocathode on pads with amplification in a single step; in this scheme, the induced signals on the pads are detected. With the use of pads and with uniforn drift aistances for all photcelectrons, the problems of high multiplicities and rates are addressed. This scheme has been demonstrated [9] to work well at low pressures ( $\approx 20$ Torr) for both CsI and Csl-TMAE photocathodes, allowing gas gains of grester than $10^{6}$ and single photoelectron efficiencies of approximately $100 \%$. In addition, there has also been significant operational experience gained with the CsI photocathode in a variety of configurations $[6,7,10]$.

One important aspect in using the C.SI and CSI-TMAE photocathodes is the use of low"pressure gas. Low pressure has been shown essential to give maximum QE [11], since noble gases at high pressure cause the photoelectrons to be scattered back into the photocathode ard thus lost. Low pressure also allows for parallel plate amplification and high gain in a single gap which is necessary for the pad readout. At low pressure, pure organic gases can be used which allow the highest gain without photon feedback. Low pressure also greatly reduces the charge deposited by passing charged particles, which have been a problerm for RICH detertors in practical applications. A minimum ionizing particle will only produce approximately 0.1 charge cluster per mm in 20 Torr of isobutane [12]. With no drift region and with parallel plate amplification the sensitive thickness of the gas is 
a few hundred microns. Thus the efficiency for detection of a charge particle directly is on the $1 \%$ level. Finally, low pressure counters offer excellent timing resolution $\{6,9,11]$.

To date there has noi been a clear picture of how to prepare and operate these photocathodes to give the maximum $\mathrm{QE}$ and the longest lifetime. The degradation of the photocathodes with operation in a wire chamber has recently received much attenion, and published results have been discouraging for their application in an high intensity environment $[4,6,9]$. There has also been a quantitative disagreement on the aging effects among different investigators. This we believe has been due to a lack of understanding of the complexity and number of processes involved. At the time of this publication some details still remain unresolved. We are in a position however, to make a substantial contribution to the understanding of the practical operation of these photocathodes, and of the key processes involved.

\section{Experimental Techniques}

Here we present enough information to make the new concepts of this work understandable without a complete review of the details of earlier works. More detail can be found in ref. 9 .

\subsection{Experimental Setup}

This research was done with several vacuum chambers similar to the one shown schematically in fig. 1. UV light enters the chamber through a quartz window. The space between the window and the photocathode is divided by stainless steel meshes into two regions: $20 \mathrm{~mm}$ thick and $1.6 \mathrm{~mm}$ thick. The $20 \mathrm{~mm}$ gap is used in determining the absolute $\mathrm{QE}$ of the photocathode with the introduction of TMAE gas as a reference. The $1.6 \mathrm{~mm}$ gap is the amplification gap above the nhotocathode.

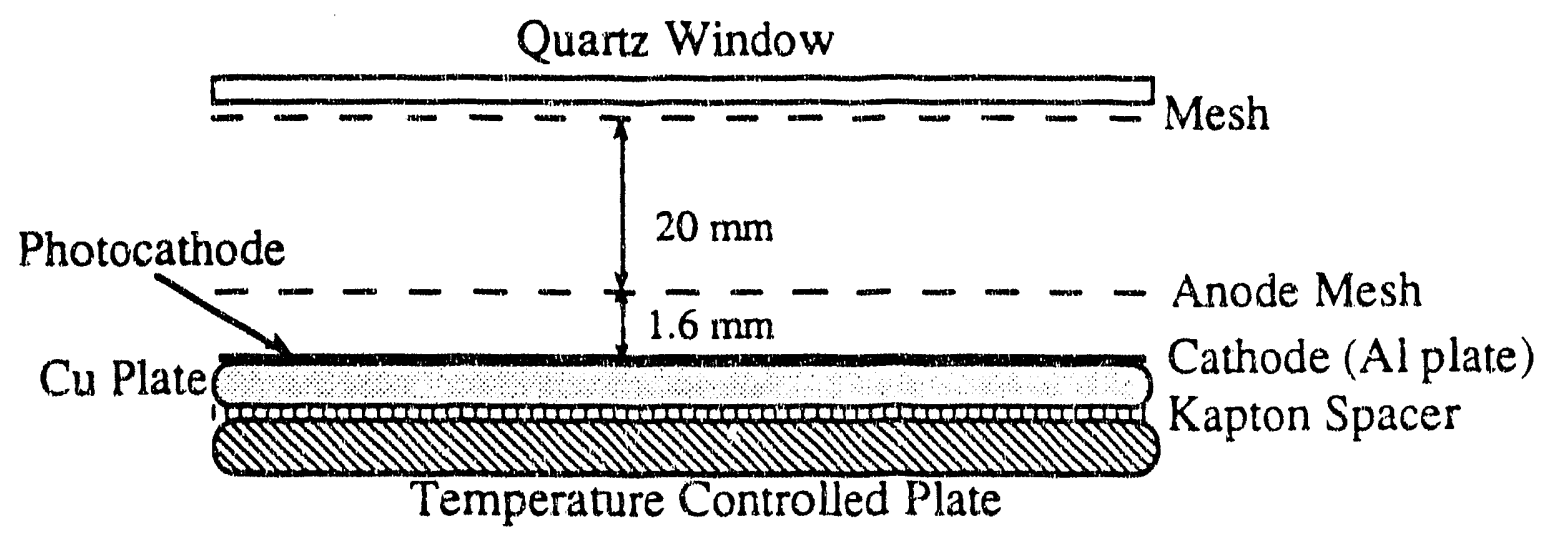

Fig. 1 Schematic of the test setup. 
The photocathode is deposited on an aluminum plate which sits on a copper plate for even heat distribution. This copper plate is electrically isolated from a second, temperature controlled plate by a kapton spacer. The temperature is either maintained by circulation of water or by a thin-film heater (e.g. Thermo/Cense, Inc. Mundelein, IL).

The gas filling pressure used as a standard was 20 Torr, usually isobutane or methane. Both the $1.6 \mathrm{~mm}$ amplification gap and the 20 Torr pressure have been determined to be about optimum from earlier studies [9]. At low pressures, we have found the best gain and stability characteristics with isobutane.

For the aging studies a DC mercury vapor lamp was used which gives emission down to approximately $170 \mathrm{~nm}$. The light was then passed through a transmission filter giving a narrow transmission at about $180 \mathrm{~nm}$. For the $\mathrm{QE}$ measurements a pulsed light source (Hamamatsu L3530-01) was used. This is a simple discharge device with a filling of approximately 100 Torr of hydrogen, wired in series with a $0.5 \mathrm{G} \Omega$ resistor and operated as a relaxation oscillator at a rate of about $60 \mathrm{~Hz}$. A pickup coil gives a trigger. This source gives a continuum spectrum with a curoff at approximately $170 \mathrm{~nm}$, and a pulse length of less than $3 \mathrm{~ns}$. The light was passed through a monochromator (Oriel model 7400) to select the desired wavelength. The monochromator was modified to be flushed with dry nitrogen to allow measurexnents down to the $170 \mathrm{~nm}$ cutoff.

\subsection{Preparation of Photocathodes}

Pieces of pure CsI scintillator are used to make the photocathodes. The CsI is vacuum deposited on an aluminum clisk to a thickness of approximately $0.23 \mathrm{mg} / \mathrm{cm}^{2}$. This thickness corresponds to $0.5 \mu \mathrm{m}$, assur.ing that the density of the photocathode is that of the solid scintillator. It has been sho'wn in a variety of works $[2,8,9]$ that there is no dependence of the CsI QE on thickness for thicknesses of $0.5 \mu \mathrm{m}$ or greater. This thickness has become our standard for these studies. Transferring and mounting the photocathode in the test chamber requires an exposure to air of about 10 minutes.

Some preliminary tests have been made with a spray-on technique. Pieces of CsI scintillator are dissolved in pure ethanol, an's the solution is sprayed on a heated aluminum plate in air. The thickness monitor is placed next to the photocathode and also heated (to drive off the alcohol) to give an estimate of the thickness. Although such photocathodes are saturated with water, heating in a vacuum (described later) restores them to a $Q E$ that approaches those made by vacuum deposition.

As will be discussed later, heating of the photocathode is a key factor in maximizing the $\mathrm{QE}$ (probably by driving off the adsorbed water). We have determined that this heating also drives off the adsorbed TMAE from a CSI-TMAE photocathode. Therefore, in 
studying that photocathode, the TMAE is no longer applied to the CsI directly after deposition in the bell jar, but later in the test chamber.

\subsection{Aging Measurements}

For the aging measurements, 8 spots on an $11 \mathrm{~cm}$ diameter, continuous photocathode were studied. Each spot is roughly $5 \mathrm{~mm}$ across with centers on a circle of 5 $\mathrm{cm}$ diameter. The center-to-center separation is thus about $2 \mathrm{~cm}$, assuring little or no effect from the stucy of adjacent spots. For a measurement of a spot, the light intensity (and, in the case of operation with charge gain, the voltage) is adjusted to give a starting value of the current of approximately $70 \mathrm{pA} / \mathrm{mm}^{2}$. The current is then automatically monitored every 10 minutes for a period of approximately 20 hours.

\subsection{Measurement of QE}

The $\mathrm{QE}$ of a photocathode is determined by comparison with the $\mathrm{QE}$ of TMAE gas. The counter is filled with 20 Torr of methane or isobutane and a sufficient voltage is applied to the anode (typically $740 \mathrm{~V}$ ) to give a plateau in the counting rate. The photocathode is operated at ground potential. The light from the pulsed light source is wavelength selected by the monochromator and the intensity is controlled by a variety of slit sizes. The intensity is reduced so that the average number of detected photons per UV pulse (trigger) is less than 0.1 at the shorter wavelengths $(\$ 190 \mathrm{~nm})$. This ensures that when a pulse is detected it represents a single photoelectron. The number of detected photons per UV pulse is then recorded as a function of wavelength.

The chamber is then evacuated and TMAE gas plus 20 Torr of methane or isobutane is added to the counter. The region between the quartz window and the "Anode Mesh" (fig. 1) is operated as a conversion and drift region with a typical collection field of $120 \mathrm{~V} / \mathrm{cm}$. For this measurement, the "Photocathode" is held at a higher positive potential (typically $740 \mathrm{~V}$ ) than the mesh marked "Anode Mesh" and is used as the true anode. This second region is operated with sufficient gain to obtain a plateau in the counting rate. This signal had no component from the solid photocathode. The number of photoelectrons detected per UV pulse is then recorded as a function of wavelength. The QE of the photocathode relative to the TMAE gas is the ratio of the number of photoelectrons per pulse for the two configurations, corrected for the optical transmission of the "Anode Mesh". The QE of the TMAE gas is taken from the literature [13], corrected for the finite absorption in the space between the quartz window and the "Anode Mesh". This yields the absolute QE of the photocathode. 
It is well known that CsI photocathodes are susceptible to moisture. Dangendorf et al. [10] have recently shown that the relative QE of a CsI photocathode can drop by as much as $50 \%$ when exposed to air for 2 hours. The flowing of very pure methane over the photocathode improves the $\mathrm{QE}$ and has in fact been employed to achieve the highest $\mathrm{QE}$ reported for CsI and CsI-TMAE [2].

We have found that the most reliable technique to maximize the $\mathrm{QE}$ is to apply heat, preferably in a vacuum [14]. Any temperature $240^{\circ} \mathrm{C}$ is sufficient to improve the photocathode. For our measurements a new photocathode is heated to $65^{\circ} \mathrm{C}$ for $15-20$ hours in a vacuum and then cooled to $25^{\circ} \mathrm{C}$. At this point TMAE is introduced if a CsITMAE photocathode is to be studied. The relatively long heating time is used to ensure the maximum $Q E$ for our measurements. Most of the benefit from heating is achieved in just a few hours.

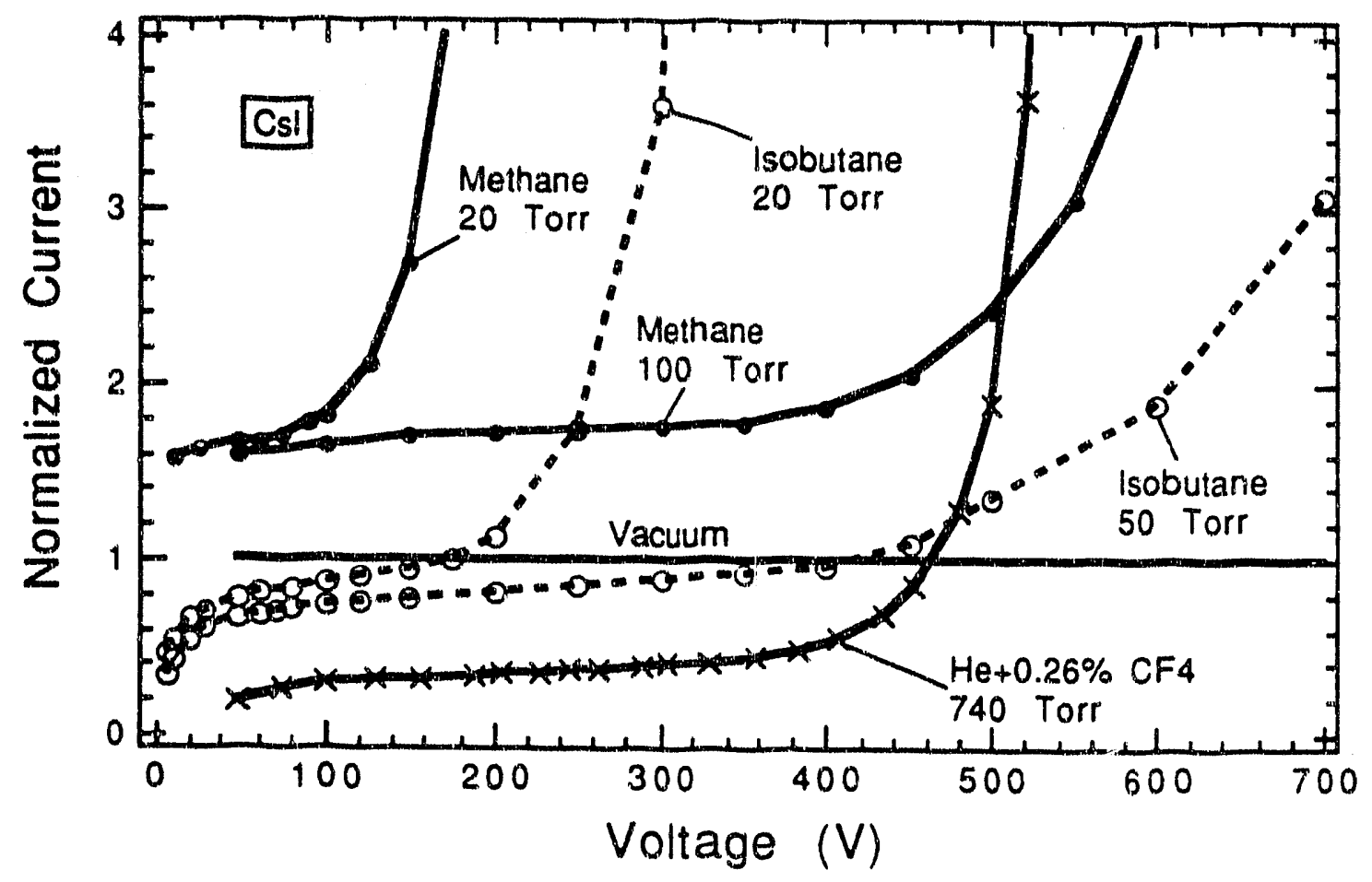

Fig. 2 Current from a CsI photocathode, normalized to the response in a vacuum, as a function of collection voltage for fillings of 20 and 100 Torr of methane, 20 and 50 Torr of isobutane, and 740 Torr of $\mathrm{He}+0.26 \% \mathrm{CF}_{4}$.

After heating, the gas environment of the photocathodes is the next most important factor in high $\mathrm{QE}$. This is demonstrated in fig. 2 which shows the current from a CSI photocathode (normalized to the response of the photocathode in a vacuum) for fillings of 
20 and 100 Torr of methane, 20 and 50 Torr of isobutane, and 740 Torr of $\mathrm{He}+0.26 \%$ $\mathrm{CF}_{4}$. The current is proportional to the QE, measured at $180 \mathrm{~nm}$. The $\mathrm{QE}$ of the photocathode in isobutane is slightly worse than the vacuum value, while that of the $\mathrm{He}$ mixture is about half the vacuum response. Methane yields a $Q E$ which approaches twice the value for vacuum. A response similar to that of methane, not shown here, was aiso measured in ethane. The reduction in the $\mathrm{QE}$ when measured in the $\mathrm{He}_{\mathrm{C}} \mathrm{CF}_{4}$ mix is well understood (see for example ref. 11): the photoelectrons are elastically scattered back to the photocathode and lost.

Fig. 3 shows the measured QE as a function of wavelength for CSI and CsI-TMAE photocathode: measured at $25^{\circ} \mathrm{C}$ in 20 Torr of isobutane and methane, and for CsI at $65^{\circ} \mathrm{C}$ measured in 20 Torr of isobutane. (The QE of CsI in methane at $65^{\circ} \mathrm{C}$ is not shown since there was not a significant difference from the result at $25^{\circ} \mathrm{C}$.) The $\mathrm{QE}$ of optically dense TMAE gas is also shown for comparison. As in fig. 2 the $Q E$ of the photocathodes in

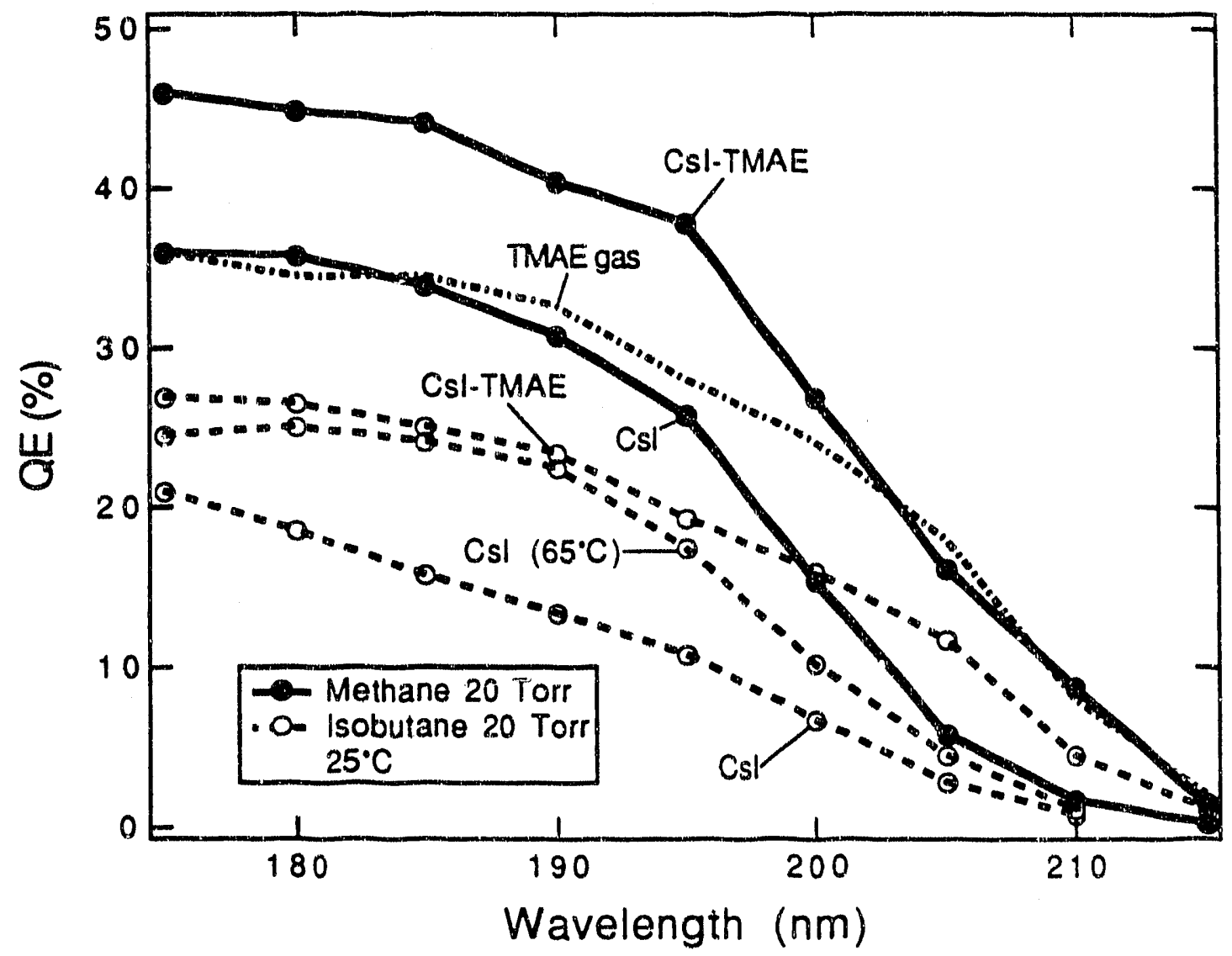

Fig. 3 Quantum efficiency as a function of wavelength for CsI and CsI-TMAE photocathodes at $25^{\circ} \mathrm{C}$ in isobutane and methane, $\mathrm{CsI}$ at $65^{\circ} \mathrm{C}$ in isobutane, and for optically dense TMAE gas. 
methane are almost a factor of 2 higher than in isobutane. The values of $Q E$, measured at $175 \mathrm{~nm}$ are $46 \%$ and $36 \%$ for CsI-TMAE and CsI, respectively. These values agree nicely with the results obtained by Séguinot et al. [2].

The difference in $\mathrm{QE}$ with gas composition is almost certainly related to adsorbed gas on the surface. (See discussion on surface phenomena ref. 15) A '-lean" surface will adsorb its first monolayer in about $1 \mu \mathrm{s}$ at a pressure of 1 Torr. The ener,;y of adsorption of the first layer is generally between 2 and $4 \mathrm{eV}$ per adsorbed atom. For additional layers, the sticking probability and binding energy drop rapidly. In the case of metals, temperatures on the order of $1000-2000 \mathrm{~K}$ are necessary to remove the last monolayer.

Heating the CsI photocathode in isobutane to $65^{\circ} \mathrm{C}$ increased the $\mathrm{QE}$ at the longer wavelengths by about $50 \%$. This is probably due to thinning of the adsorbed layer by heating. The effect is most pronounced at longer wavelengths where the energy of the photoelectron is lowest.

We have demonstrated the importance of surface gases with stainless steel and aluminum photocathodes. With a variety of gases, the QE at $180 \mathrm{~nm}$ increased with pressure, saturating at a pressure for all gases at about 1 Torr. For pressures of 1 Torr or greater, the $\mathrm{QE}$ was a factor of two higher than the value in a vacuum. Since the metals are not very absorbent for the gases, this would seem to be a surface, rathe: than a bulk, phenomenon. Heating of these metal photocathodes also increase their $Q E$.

In the work of Séguinot et al. [2] they obtained their high values of $Q E$ after flushing their photocathode with methane while maintaining a temperature of $40^{\circ} \mathrm{C}$ [3]. Their $\mathrm{QE}$ measurements were made with methane. Both factors are now believed to be crucial to obtaining the maximum response. The early work by Kwan and Anderson [4] that measured a similar value fo: he CSI-TMAE photocathode obtained this result with methane after the collection of a substantial charge from the photocathode. We now feel that the current from the photocathode caused local heating enhancing it much as we do now by other means.

\section{$4 \quad$ Aging Results}

The work that is presented in this section consist of many data sets with over 100 data points each. Fig. 4 is a representative data set showing the current as a function of collected charge for a CsI photocathode aged in 20 Torr of isobutane. The fit to the data, as well as the data points are shown. Since many of the figures in this section will be comparisons of several measurements, the data points are omitted for clarity and only the fits to the data shown. Since all starting vurrents are similar in value (approximately $70 \mathrm{pA} / \mathrm{mm}^{2}$ ), whe a several fits are compared in a single figure they are scaled to a starting value of 100 to facilitate comparisons. 


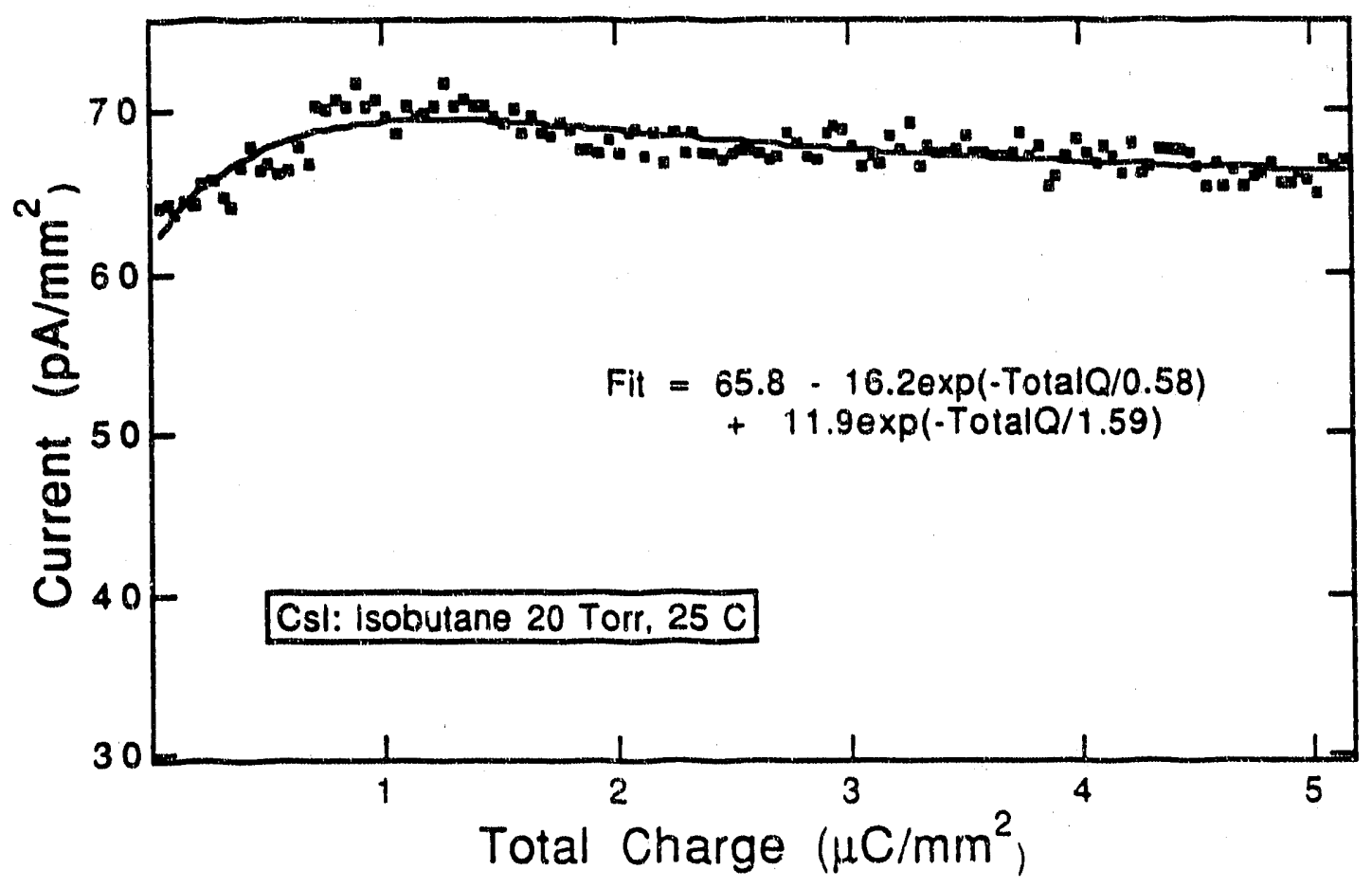

Fig. 4 Current as a function of total collected charge for a CsI photocathode aged in 20 Torr of isobutane at $25^{\circ} \mathrm{C}$.

We know that methane or ethane give the best $\mathrm{QE}$ for the photocathodes that we are studying here. This is a recent discovery, and so most of the aging measurements are made with isobutane, which was chosen because it gives the highest gain and the best stability.

It should be stressed that, although measurements made on a single photocathode deposition are reproducible, there is a cathode-to-cathode variation in the results. The largest variations are seen in the vacuum i leasurements. As an example, for aging measurements made in a vacuum, the $\mathrm{QE}$ might vary from $15 \%-30 \%$ of the initial value for a total collected charge of $3 \mu \mathrm{C} / \mathrm{mm}^{2}$. We have yet to determine which variable is not being adequately controlled, but in general what we present here is true for all photocathodes that we have studied.

\subsection{Effects of Gas on Aging}

We have found a great improvement in the aging characteristics of the CsI photocathode, and to a lesser extent of the CsI-TMAE photocathode, when operated in the presence of a gas. Fig. 5 shows the normalized current as a function of total collected charge for CSI and CSI-TMAE photocathodes aged in vacuum and in 20 Torr of isobutane. The most obvious point of this figure is that the addition of the isobutane made a small 
improvement to the aging properties of the CsI-TMAE photocathode but a much more dramatic improvement for pure CsI.

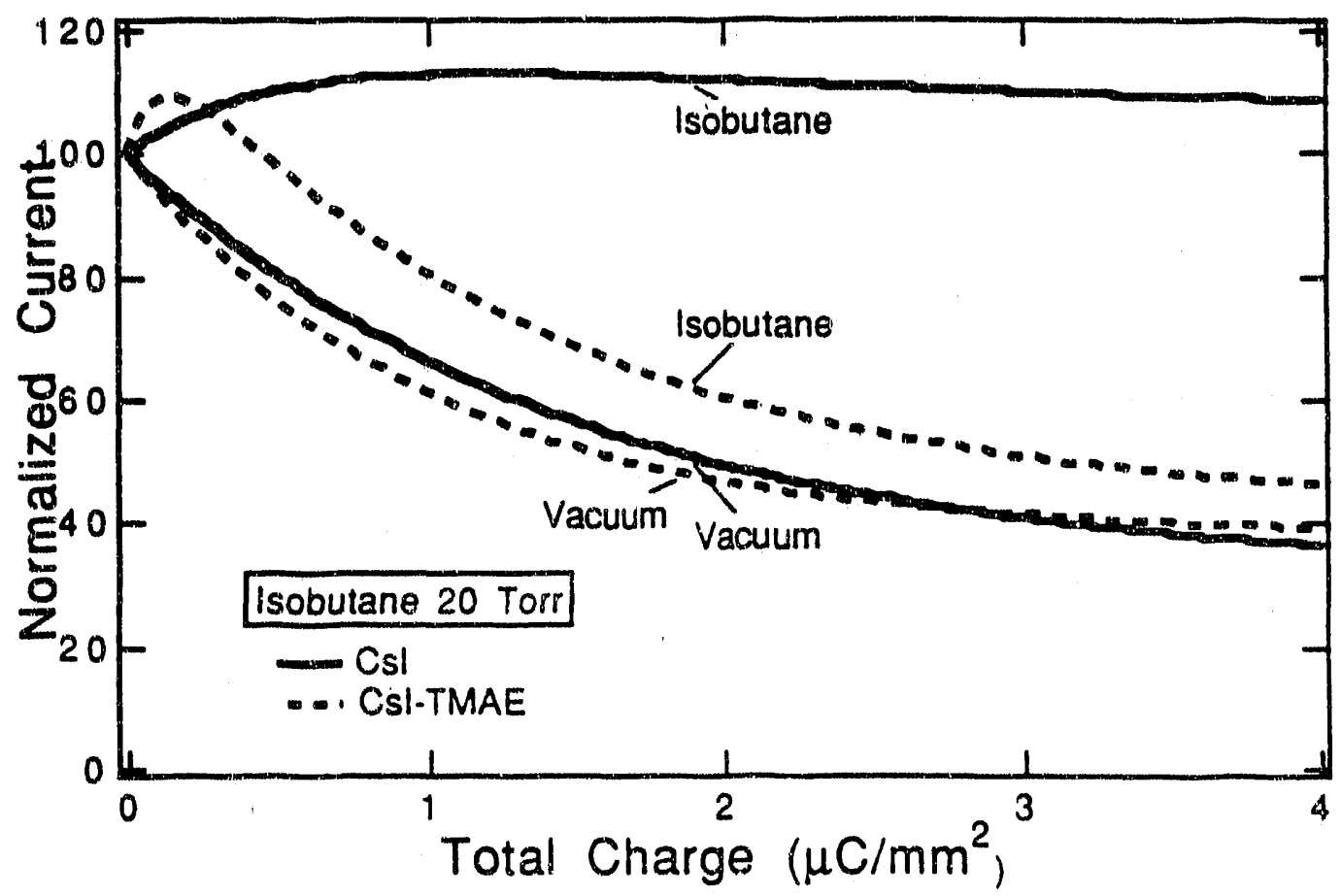

Fig. 5 Normalized current as a function of total collected charge for CsI and CsI-TMAE photocathodes aged at $25^{\circ} \mathrm{C}$ in vacuum and in $20 \mathrm{Torr}$ of isobutane.

Another, perhaps more subtle, difference in the aging characteristics of these photocathodes is that with the addition of gas the output first increases with time. We found this to be characteristic of almost all our measurements made with various gases and believe it to represent an actual increase in $\mathrm{QE}$. The equation that we fit to such data is:

$$
\text { Current }=k_{1}-k_{2} \exp \left(- \text { Total } Q / k_{3}\right)+k_{4} \exp \left(- \text { Total } Q / k_{5}\right) \text {, }
$$

where $\mathrm{k}_{\mathrm{i}}$ are constants, and TotalQ is the total collected charge.

Fig. 6 shows the normalized current as a function of total collected charge collected for a CsI photocathode in a vacuum and 4 different pressures of isobutane. One can see that the effect of gas increases with pressure. By 20 Torr the effect of aging is very small and at 100 Torr the $\mathrm{QE}$ seems to increase linearly with collected charge.

Fig. 7 shows the current as a function of total charge for a CsI photocathode operated at a high current in 20 Torr of isobutane. The starting current is $600 \mathrm{pA} / \mathrm{mm}^{2}$. After an accumulated charge of over $30 \mu \mathrm{C} / \mathrm{mm}^{2}$, the $\mathrm{QE}$ had only dropped by $35 \%$. This 


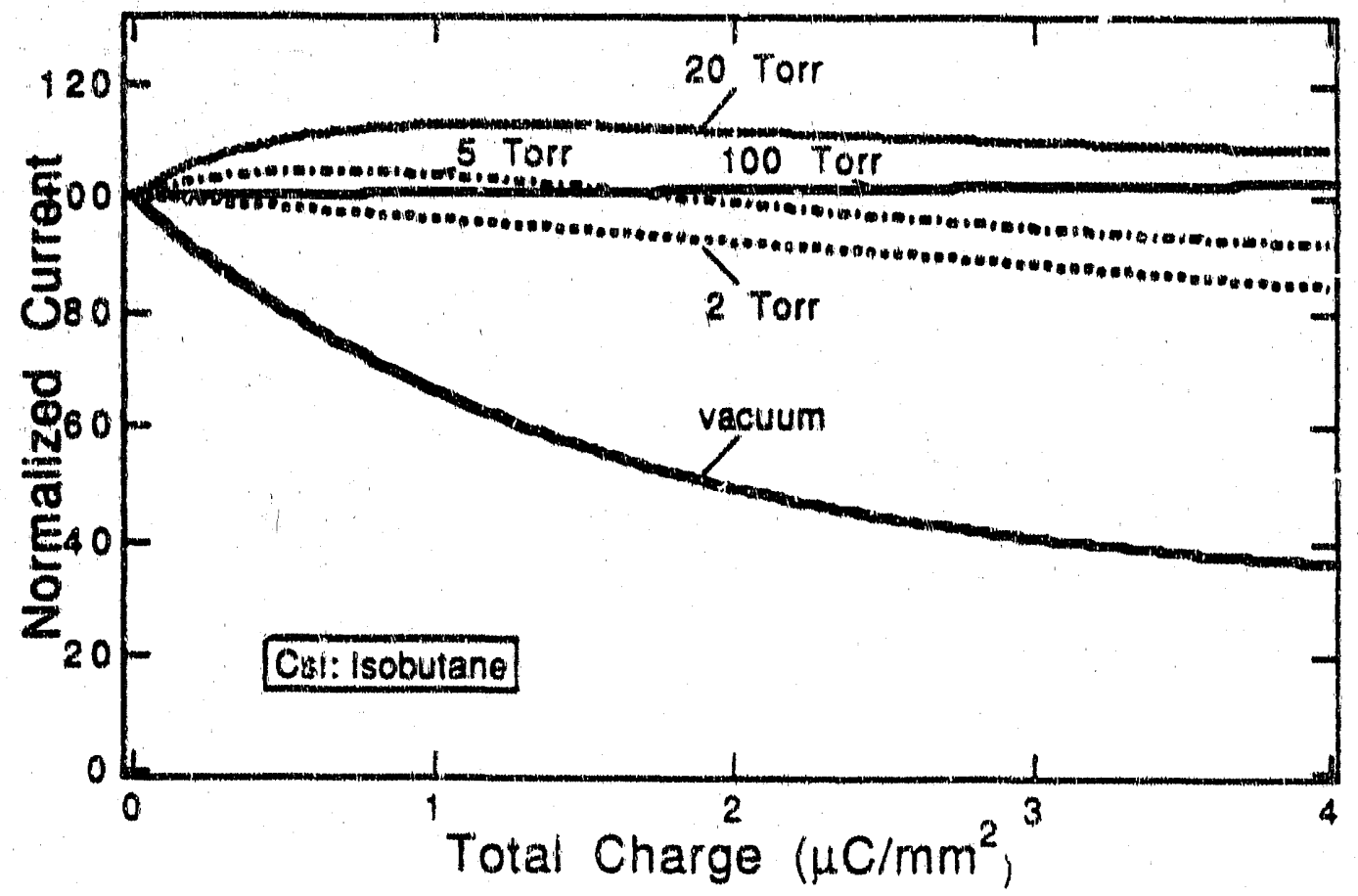

Fig. 6 Normalized current as a function of total collected charge for CsI photocathodes aged in vacuum and 4 different pressures of isobutane.

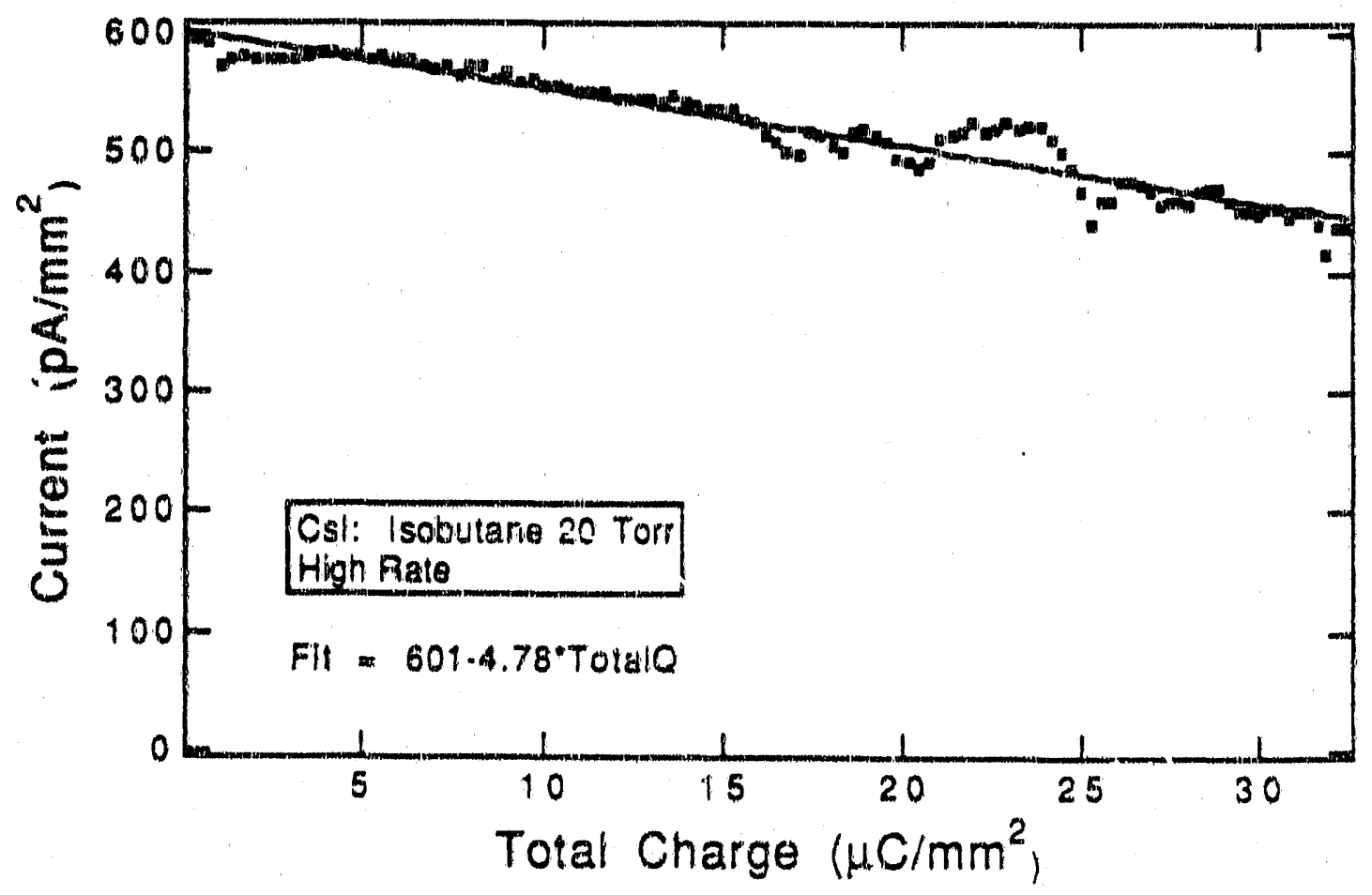

Fig. 7 Current as a function of total collected charge for a CsI photocathode aged at a high rate in 20 Torr of isobutare at $25^{\circ} \mathrm{C}$. 
accumulated charge corresponds to over $2 \times 10^{14} \mathrm{e} / \mathrm{mm}^{2}$. If the charge were collected at a lower rate and if the pressure were somewhat higher even less degradation is expected.

The normalized current as a function of total charge for a variety of gases is showr in fig. 8. We believe that aging of the CsI photocathode in a vacuum is due to dissociation of the CsI with the liberarion of iodine leaving cesium metal (discussed below). We assume that the improvement in the aging characteristics of the CsI photocathode in gas is due to adsarption of the gas on the surface or in the bulk which somehow prevented the dissociation. As can be seen in fig. 8, argon also improved the performance of the photocathode. Even noble gases are believed to be adsorbed to the extent of a monolayer or two [15].

\subsection{Effects of Temperature on Aging (CsI Only)}

In the case of CsI photocathodes, we were able to investigate the effects of temperature on aging. This was not the case with the Csi-TMAE photocathode since heating caused the TMAE to be removed from the surface. Fig. 9 shows the normalized

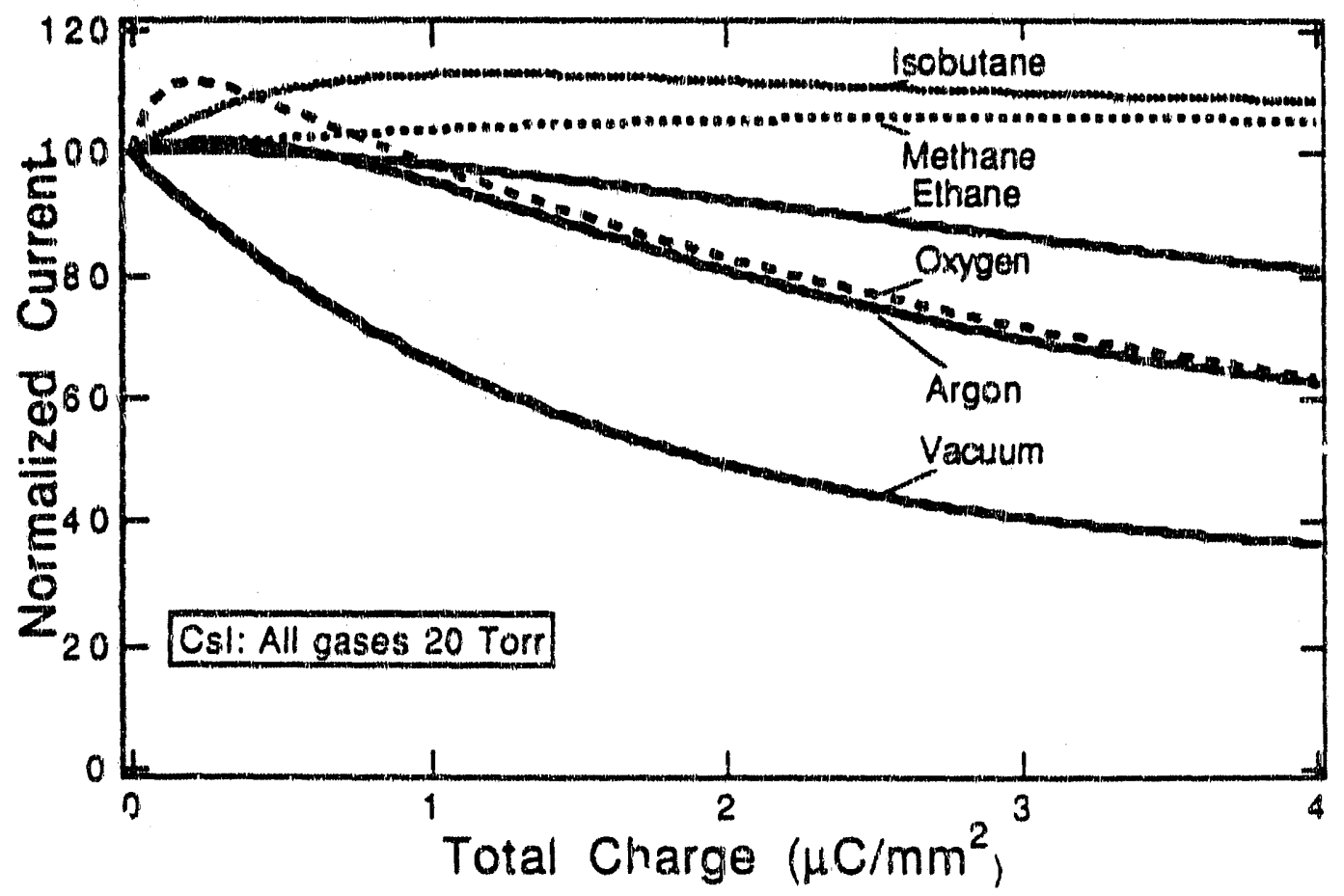

Fig. 8 Nommalized current as a function of total collected charge for CsI photocathodes aged in vacuum and in 5 different gases at 20 Torr.

current as a function of total collected charge for CsI photocathodes in vacuum and 20 Torr of isobutane measured at $25^{\circ} \mathrm{C}$ and $65^{\circ} \mathrm{C}$. In both vacuum and gas the higher temperature 
has a better aging characteristic. The $65^{\circ} \mathrm{C}$ measurement with isobutane continues to increase with accurnulated charge. This is perplexing but seen in other measurements.

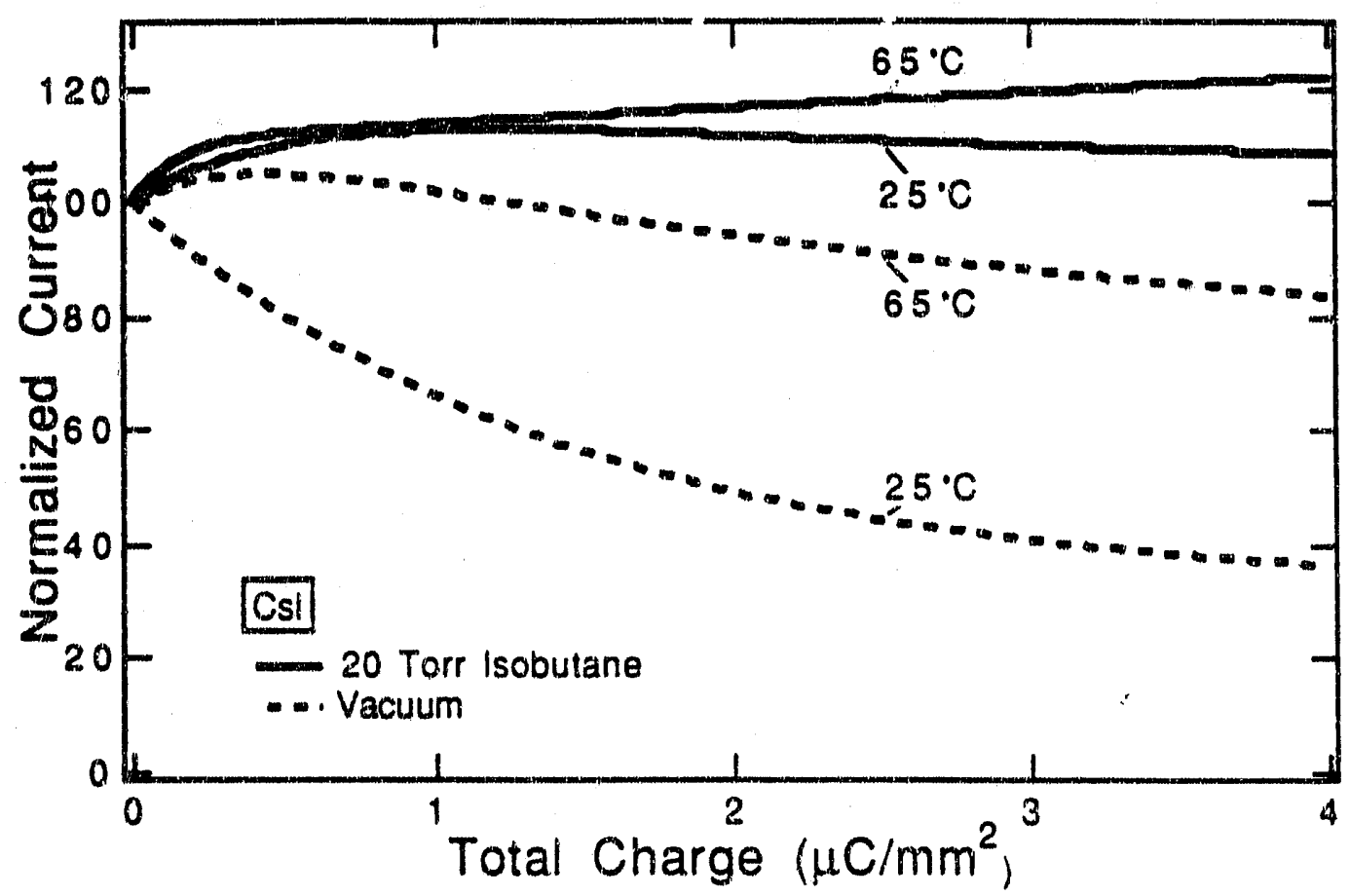

Fig. 9 Normalized current as a function of total collected charge for CsI photocathodes aged in vacuum and 20 Torr of isobutane at $25^{\circ} \mathrm{C}$ and $65^{\circ} \mathrm{C}$.

A nice demonstration of the dependence of both $\mathrm{QE}$ and aging on temperature is shown in fig. 10. Here a CsI photocathode was aged in a vacuum. The temperature of the photocathode was maintained at $25^{\circ} \mathrm{C}$ until time $\mathrm{t}_{1}$ at which time the temperature was increased to $65^{\circ} \mathrm{C}$. From $\mathrm{t}_{2}-\mathrm{t}_{3}$ the UV light source was blocked between measurements. After time $t_{3}$ the UV light source was again left unblocked and the photocathode cooled to $25^{\circ} \mathrm{C}$. The $\mathrm{QE}$ dependence on the temperature is obvious. On cooling the current did not return to the line fitted to the earlier data points. There has been some recovery.

\subsection{Effects of Gas Gain on Aging}

With a better understanding of the variables involved in aging, more reproducible measurements have become possible. We have confirmed what was seen elsewhere [5], that the positive ions do not have the same effect as UV photons. Fig. 11 shows the normalized current of CsI photocathodes as a function of total collected charge for gas gains of 1 and $3.5 \times 10^{4}$. The difference in response is apparent. Unforunately, we can 
only speculate as to the reason for the $20 \%$ increase in current for the high-gain measurement.

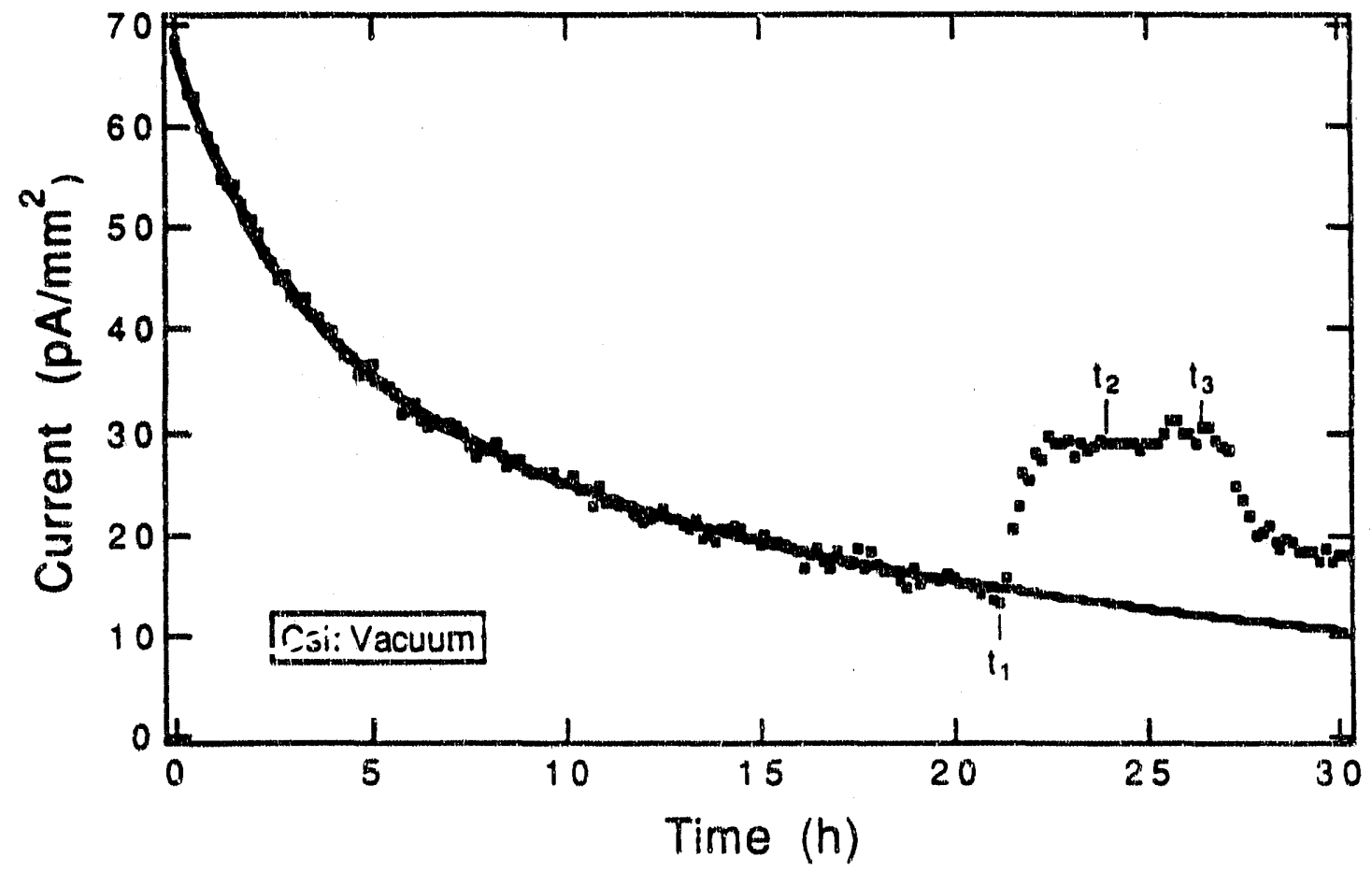

Fig. 10 Current as a function of tine for a CsI photocathode aged in a vacuum. At $t_{1}$ the temperature is increased from $25^{\circ} \mathrm{C}$ to $65^{\circ} \mathrm{C}$. From $\mathrm{t}_{2}$ to $\mathrm{t}_{3}$ the UV light is blocked between measurements. At $t_{3}$ the light is unblocked and the temperature returned to $25^{\circ} \mathrm{C}$.

Fig. 11 also shows the normalized current of CsI-TMAE photocathodes as a function of charge for gas gains of 1,40 , and 350 . The higher the gain, the lower the light intensity used. From these curves, one can see that the degradation of the photocathode by the positive gas ions is less than that caused by the loss of the photoelectrons. Thus the aging predicted by vacuum measurement of the CSI-TMAE photocathode is greater than what would be expected for a real device operated with substantial gas gain.

\subsection{Discussion of Aging Results}

We think that we may have an understanding of the behavior of CSI in a vacuum. The cesium and iodine atoms are joined by an ionic bond. When the photoelectron is removed the bond is lost. Since iodine has a substantial vapor pressure, the iodine is lost to the vacuum. This leaves residual cesium metal as well as creating vacancies and $F$-centers in the crystal lattice [16]. The result :s a lower $Q E$ but an increased sensitivity to room light because of the lower photoionization threshold of cesium and F-centers. Since cesium 
metal also has a non-zero vapor pressure, it also leaves the photocathode (if no oxygen is present). Thus the aging characteristic of the photocathode in vacuum is dependent on the rate and

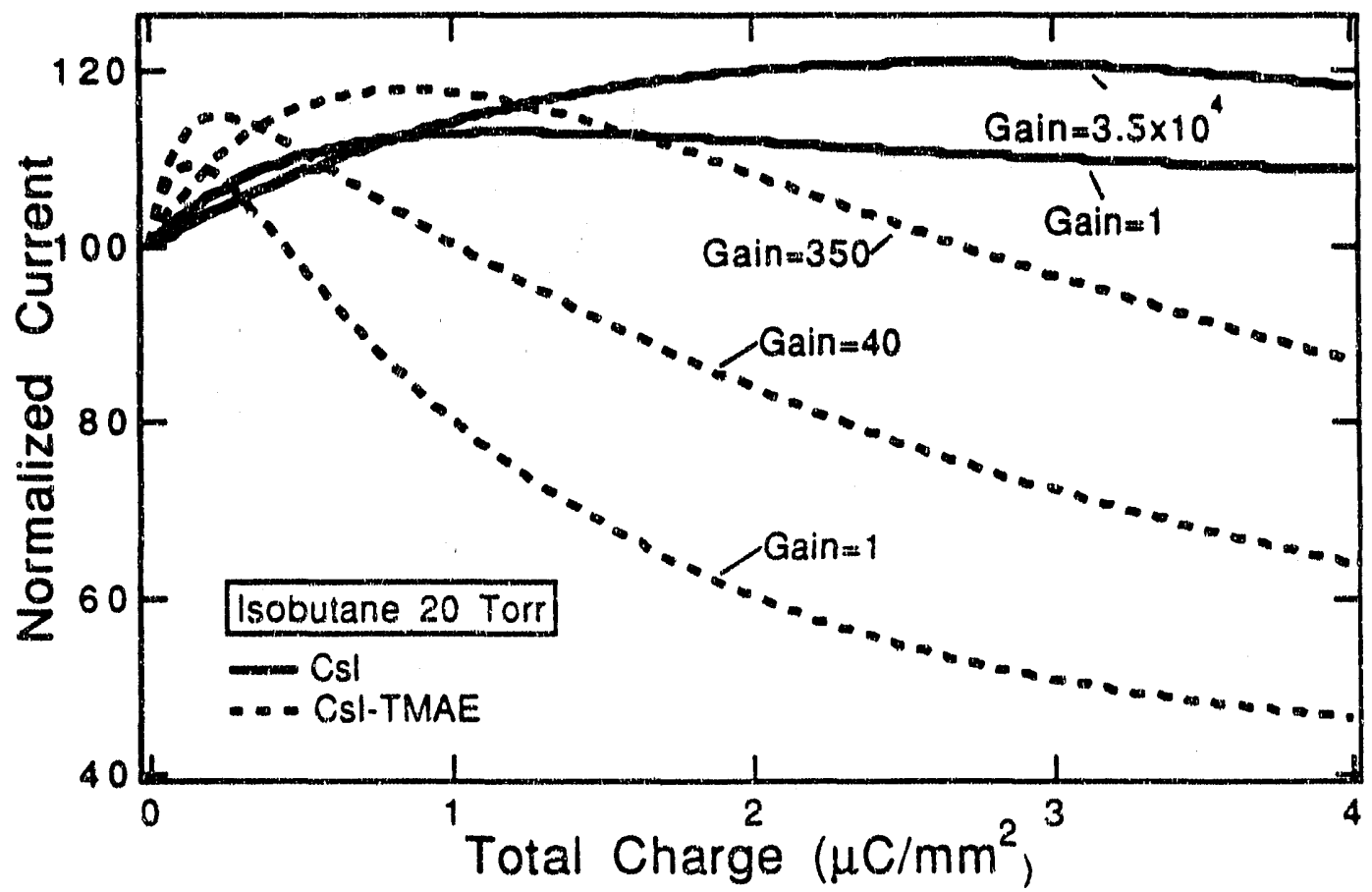

Fig. 11 Normalized current as a function of total collected charge for CsI and CsI-TMAE photocathodes aged in 20 Torr of isobutane at a variety of charge gains.

temperature. The recovery seen in fig. 10 is believed to be due to increased evaporation of the cesium at the higher temperature.

When left in a good vacuum, a CsI photocathode will almost completely recover. In a demonstration of this effect, a very thick photocathode was prepared. One-half of the photocathode was then exposed to a very intense UV lamp in a vacuum. After a large exposure the photocathode was then allowed to remain in the vacuum for an additional week. The photocathode was then removed and examined with a scanning electron microscope. The exposed part had almost all of the CsI removed but the ratio of cesium to iodine remained essentially the same on both halves.

The effect of gas on the aging of the CsI photocathode is more difficult to understand. The presence of gas somehow inhibits the dissociation of the CsI when the photoelectron is removed.

In the case of the CSI-TMAE photocathode in vacuum, much of the damage is due to dissociation of the TMAE on the surface. For the CsI-TMAE photocathode in gas, litule improvement is seen (at gain=1) since the surface is already saturated with TMAE, and the 
aging is again believed to be due to degradation of the TMAE. The positive ions do not cause the same damage to the photocathode as the removal of the photoelectrons.

At least in the case of the CsI photocathode operated in gas, total extracted charges of over $30 \mu \mathrm{C} / \mathrm{mm}^{2}\left(2 \times 10^{14} \mathrm{e}^{-/ \mathrm{mm}^{2}}\right)$ can be achieved with little damage to the photocathode. This was obtained at very high currents. There are indications that at lower rates there is even less aging. These measurements will be repeated for methane which also appears to offer good aging characteristics and better $Q E$.

\section{$5 \quad$ Single-Electron Timing Resolution}

Because the low pressure chamber with a solid photocathode amplifies directly above the photocathode and in a very small volume, it is assumed that the timing characteristics will be very good. We hope to combine such a chamber with a solid or liquid Cherenkov radiator to produce a time-of-flight counter.

We have made a first attempt to demonstrate a time-of-flight counter based on a CsI-TMAE photocathode. Two chambers, similar to the one shown schematically in fig. 12, were each constructed with $4 \mathrm{~cm}$ diameter photocathode, and a $1.6 \mathrm{~mm}$ cathode to anode mesh spacing. The radiators for the chainbers were $1.5 \mathrm{~cm}$ thick cells filled with perfluorohexane $\left(\mathrm{CF}_{3}\left(\mathrm{CF}_{2}\right)_{4} \mathrm{CF}_{3}\right)$. The gas filling was 20 torr of isobutane, with an

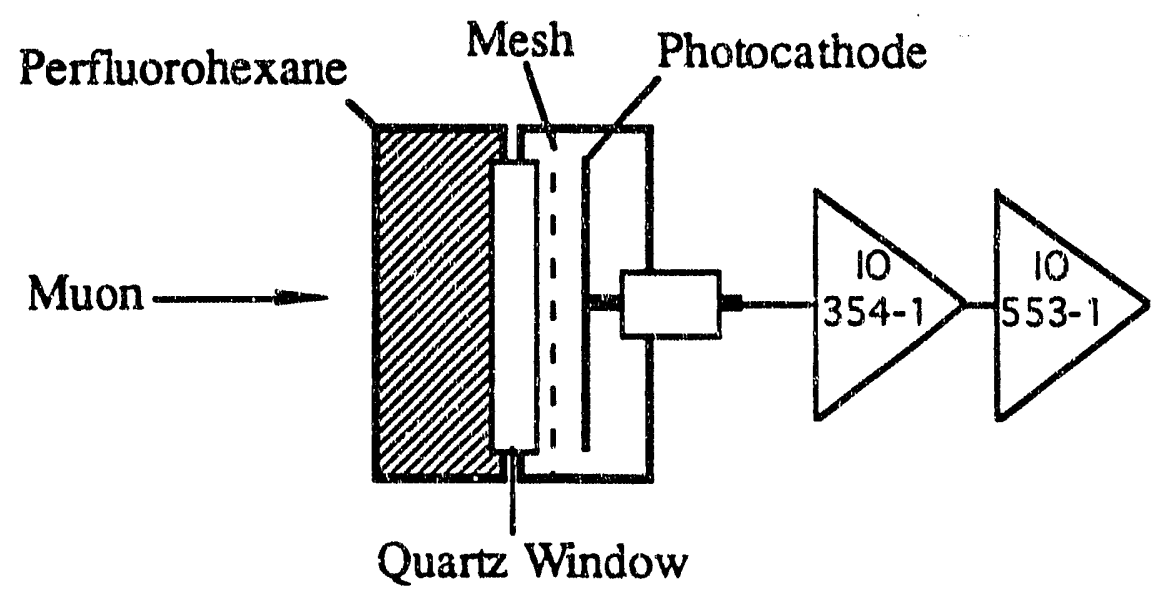

Fig. 12 Schematic of the test chambers used in the time-of-flight measurements.

operating voltage of $980 \mathrm{~V}$ and a gain of approximately $10^{7}$. The chambers had a very simple design. We were not able to heat the photocathode after the perfluorohexane cell was connected, and we were not able to flow the gas. We also had problems with the purity of the perfluorohexane as well as a geometric problem for light collection for incident particles off axis. This combination yielded a measured probability of about $50 \%$ that a muon passing through the chamber would produce a single photoelectron. 
The electronics for the beam test were developed by Radeka's group at Brookhaven National Laboratory. The preamplifiers (IO-354-1) were charge sensitive amplifiers with a $1 \mathrm{~ns}$ rise time and a 40-60 ns fall time, while the shaping amplifiers (10-553-1) were double pole-zero shaping amplifiers with a $3 \mathrm{~ns}$ shaping time. This combination gave a signal for a single photoelectron with a width of less than $20 \mathrm{~ns}$ and an amplitude of approximately $30 \mathrm{mV}$. (Both amplifiers are described in ref. 12.) The shaping amplifiers were followed by constant fraction discriminators.

Fig. 13 shows the timing spectrum from a muon test made at Fermilab with the two chambers. One chamber was used for the start and the other for the stop. The total singlephotoelectron resolution is $0.77 \mathrm{~ns}(1 \sigma)$. If it is assumed that both chambers contribute equally to the resolution, this would imply a timng resolution for a single chamber of $0.55 \mathrm{~ns}$.

These are encouraging results. With properly designed chambers, and clean perfluorohexane, one would expect many more photoelectrons. This would yield a timeof-flight counter with an excellent timing resolution, low mass $(\leq 0.2 \mathrm{Xo})$, and good granularity.

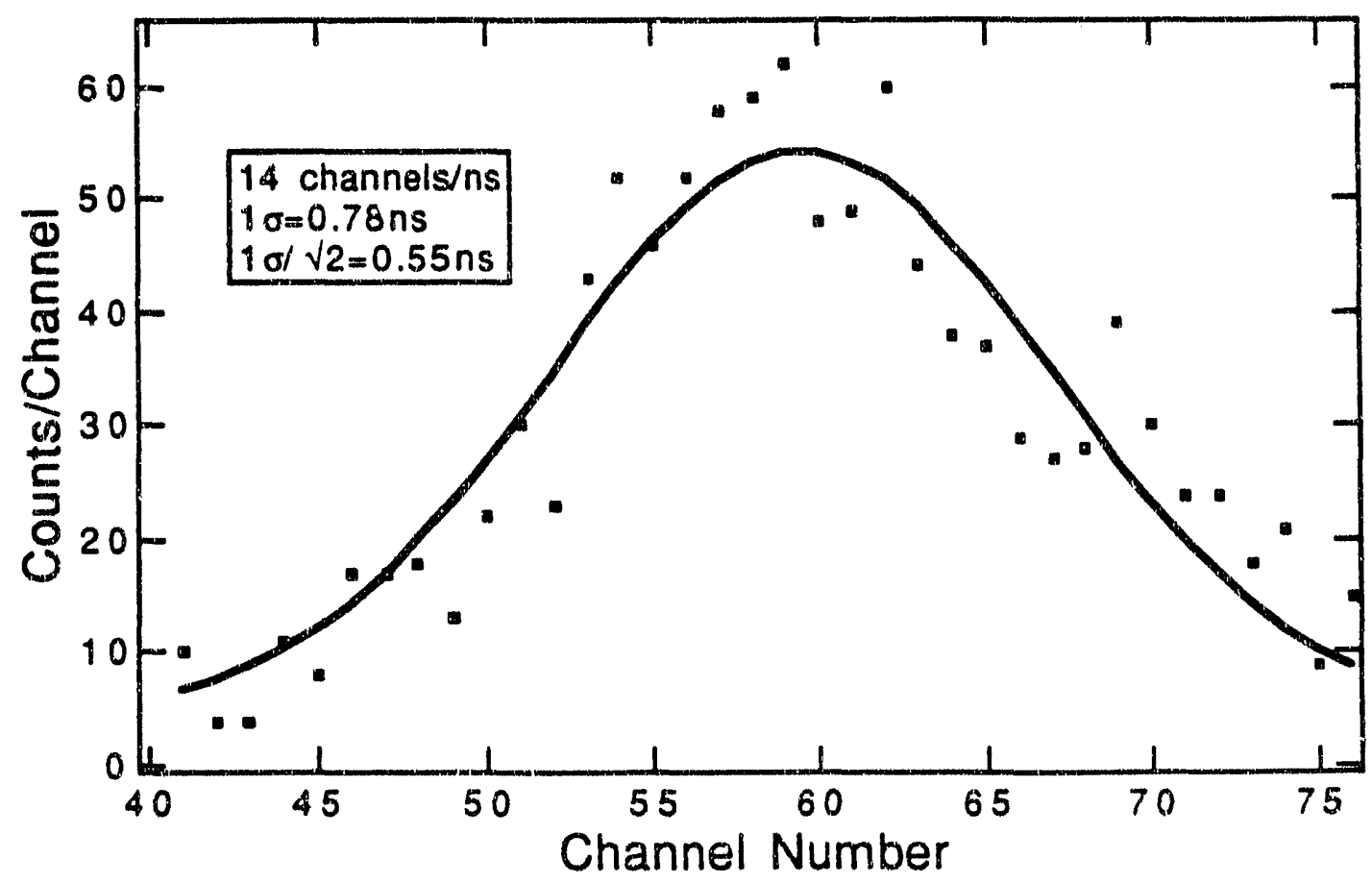

Fig. 13 Single electron timing spectrum for muons made with 2 low-pressure chambers. 
Summary and Conclusions

The key factors in obtaining a reproducibly high QE for the CSI and CsI-TMAE photocathodes are: 1) heating to a temperature of $240^{\circ} \mathrm{C}$ in a vacuurn or with a flow of a suitable gas, and 2) operating the photocathode in the proper gas such as methane. The heating is believed to remove the adsorbed water, and possibly other gases. In a practical detector, the heat can be applied by a thin-film heater placed directly under the photocathode.

The use of a gas such as methane yields a QE nearly a factor of 2 higher than for isobutane and nearly a factor of 4 higher than for $\mathrm{He}+0.26 \% \mathrm{CF}_{4}$. This effect is believed to be related to adsorption of gas on the surface of the photocathode.

Aging of the photocathodes is shown to depend on the pressure and type of gas present, as well as the temperature. The amount of gas gain is also important since, in the case of the CsI-TMAE photocathode, the positive ions do not do as much damage as the removal of an electron by a photon. At least in the case of the CsI photocathode operated in isobutane, total collected charges of $2 \times 10^{14} \mathrm{e}^{-/} \mathrm{mm}^{2}$ or greater will not degrade the performance by more than a few per cent. Since the bulk CsI has been shown to keep its scintillation and transmission characteristics after doses on the Mrad level [17], this photocathode should survive in an SSC/LHC hadron environment. These photocathodes can also handle the rates of such an environment, since the shaped pulse can be made to be less than $20 \mathrm{~ns}$ and pad readout allows for high multiplicities.

One concern when considering the low pressure operation is the question of how to construct an instrument with a large area. With the single-step amplification described in this work, one can conceive of supporting the window and the back plane off each other with two G-10 or ceramic support grids, above and below the anode mesh. Thus, the distance that the window and back plane must span without support is only $5 \mathrm{~cm}$ or so. Since low pressure operation requires only low voltages, sparking down the insulator is no longer a problem.

This work is far from completed. A greater understanding must be gained of the remaining variables. Further work must also be done on the "spray on" technique which, if it proves to produce damage resistant photocathodes, promises to be a useful technique for covering large areas. The effect of adsorbed gas also merits further study, particularly the study of aging of the photocathode in a methane environment. There is also the hope that a gas can be found which will lower the work function of the surface.

There is much work to be done on using these photocathodes, coupled to lowpressure counters and liquid or solid Cherenkov radiators for time-of-flight detectors. They may yield large-area instruments with good timing resolution and granularity. 
We believe that the quantum efficiencies presented in this work are routinely achievable. The techniques for preparation and operation are accessible to those with normal instrumental skills. The CsI photocathode and possible the CsI-TMAE photocathode are ready for inclusion in a high energy physics experiment. The results sliould be instruments with good $\mathrm{QE}$, excellent timing, able to work at high rates and high multiplicity, and which are insensitive to passing particles.

\section{Acknowledgments}

The authors would like to express their gratitude to Rohert W. Sparro'n (Optovac, Inc.) for many useful discussions as well as his material support of our measurements. We would like to thank Veljko Radeka an 1 Craig Woody for assistance with the electronics and for valuable discussions. We would aiso like to thank Hobie Kraner for assistance with the electronics and for making the analysis with the scanning-electron microscope pcssible.

\section{References}

[1] J. Va'vra, IEEE Trans. Nucl. Sci, NS-34 (1987) 486; C.L. Woody, IEEE Trans. Nucl. Sci, NS-35 (1988) 493.

[2] J. Séguinot et al., Nucl. Instr. and Meth. A297 (1991) 133.

[3] V. Peskov, private communications.

[4] S. Kwan and D.F. Anderson, Nucl. Instr. and Meth. A309 (1991) 190.

[5] G. Charpak, V. Peskov and D. Scigocki, Studies of BaF ${ }_{2}$ Calorimeters in Future Hadron Colliders, talk given at the 8th Workshop of the INFN-ELOISATRON Project on Perspectives for New Detectors in Future Supercolliders, Erice, 17-24 Oct. 1989.

[6] V. Dangendorf, et al., Nucl. Instr. and Meth. A308 (1991) 519.

[7] B. Guerard, et al., Nucl. Instr. and Meth. A310 (1991) 116.

[8] D.C. Imrie, et al., Nucl. Instr. and Meth. A310 (1991) 122.

[9] H. Hoeneisen, D.F. Anderson, and S. Kwan, Nucl. Instr. and Meth. A302 (1991) 447.

[10] V. Dangendorf, A. Breskin, R. Chechik and H. S hmidt-Bocking, Nucl. Instr. and Meth. A289 (1990) 322.

[1i] D.F. Anderson et al., Nucl. Instr. and Meth. 217 (1983) 217.

[12] J. Fischer, et al., Nucl. Instr. and Meth. A238 (1985) 249. 
[13] R.A. Holroyd, J.M. Preses, C.L. Woody and R.A. Johnson, Nucl. Instr. and Meth. A261 (1987) 440.

[14] D.F. Anderson, et al.,Technical Memo on New Results on CsI Photocathodes: Enhancement and Aging, Fermilab TM-1753, September 1991.

[15] E.W. McDaniel, Collision Phenomena in Ionized Gases, (Wiley, New York, 1964). p.626.

[16] A.J. Dekker, Solid State Physics, (Prentice-Hall, Eaglewood Cliffs, 1962) p.366.

[17] C.L. Woody et al., "Radiation Damage in Undoped CsI and CsI(Tl)", to be published in IEEE Trans. Nucl. Sci., NS-39 (1992). 

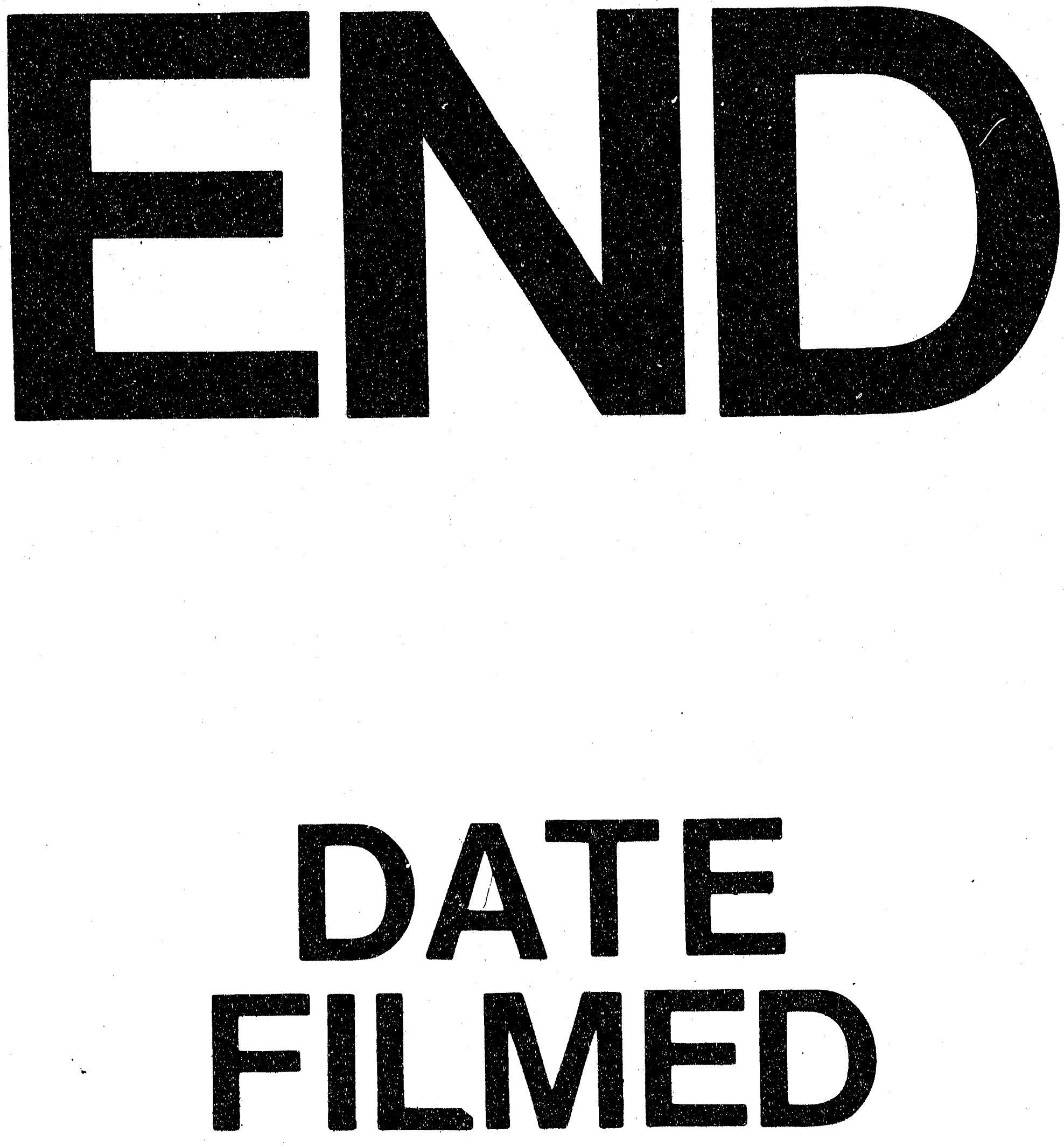

I

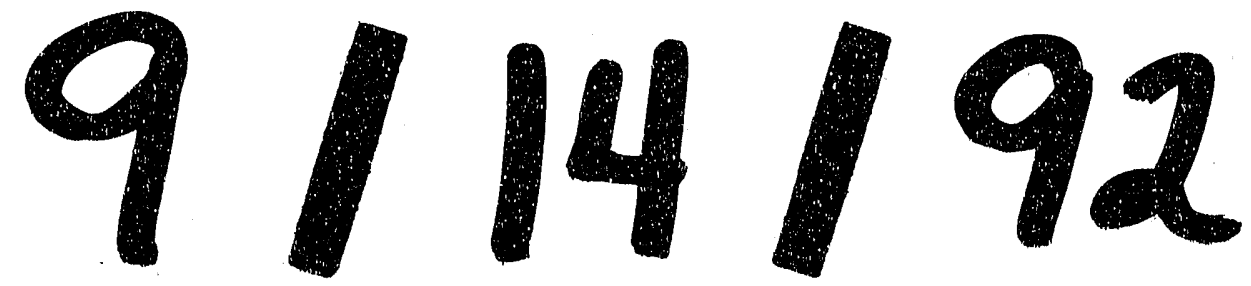


